\title{
Grupo de Estudo, Pesquisa e Extensão em Robótica e Automação Como Fator Motivacional Para Estudantes de Computação
}

\author{
Matheus E. Franco' ${ }^{1}$, Breno M. Barra ${ }^{1}$, Rosana A. Moreira ${ }^{1}$, Caio C. Dias ${ }^{1}$ \\ ${ }^{1}$ Departamento de Computação - IFSULDEMINAS- Campus Machado \\ CEP - 37.750-000 - MG - Brasil \\ matheus.franco@ifsuldeminas.edu.br, brenno barra@hotmail.com, \\ rooh.moreiraalgmail.com, caiodias910@gmail.com
}

\begin{abstract}
In Computer courses the difficulties encountered by students and the high dropout rates are notorious. Thus, it is necessary to develop activities that privilege the contemporary scenario with the objective of motivating and externalizing the students' creativity. This paper describes the creation and implementation of a study, research and extension group in robotics and automation using the Arduino and the LEGO Mindstorms robotics kit. The results show that the proposal can be an interesting resource to develop in academics computational communities in view of the high agreement obtained.
\end{abstract}

Resumo. Nos cursos de computação são notórias as dificuldades encontradas pelos estudantes e as altas taxas de evasão. Assim, é necessário o desenvolvimento de atividades que privilegiem o cenário contemporâneo com o objetivo de motivar e externar a criatividade dos estudantes. Este trabalho descreve a criação e implantação de um grupo de estudo, pesquisa e extensão em robótica e automação utilizando a plataforma Arduino e o Kit de robótica LEGO Mindstorms. Os resultados obtidos demonstram que a proposta pode ser um recurso interessante a se desenvolver nas comunidades acadêmicas de computação tendo em vista a alta concordância obtida.

\section{Introdução}

A partir dos anos 2000 vivenciamos uma expansão considerável na oferta de cursos de Computação no Brasil, seja de nível médio ou superior [INEP 2014]. Apesar deste crescimento, o índice de evasão em cursos superiores da área chega a ultrapassar $80 \%$ [Hoed 2017], sendo que dentre os diferentes fatores que influenciam a evasão, encontrase a desmotivação dos alunos em razão do estilo de ensino utilizado e a aprendizagem esperada [Csikszentmihalyi and Wong 2014].

Este cenário possui grande relevância, pois estudos encomendados pela Cisco apontam um déficit superior a 195 mil profissionais na área de computação somente no Brasil em 2015 [Cisco 2016]. Desta maneira, considerando a evolução da robótica, automação e das tecnologias de informação e comunicação, o contexto educacional do ensino de computação exige transformações visando o desenvolvimento de novos métodos que privilegiem o cenário do mundo contemporâneo. Torna-se necessário 
desenvolver atividades que motivem e instiguem os alunos a aprender novas habilidades, assimilar novos conceitos, avaliar novas situações, lidar com o inesperado.

A realização de diferentes atividades é necessária, pois o processo de aprendizagem não é vivenciado por todos os estudantes da mesma maneira, sendo que cada aluno pode desenvolver um diferente estilo de aprendizagem enfatizando algumas habilidades sobre as outras [Felder and Silverman 1988]. Uma vez que os alunos possuem diferentes estilos de aprendizagem, é interessante desenvolver métodos e atividades com características diferenciadas utilizando variados formatos além dos tradicionais expositivistas ministrados em sala de aula.

Neste contexto, a realização de atividades multidisciplinares que envolvam computação, automação e robótica é uma prática consolidada, sendo que trabalhos recentes relatam experiências nesta vertente [Correll et al. 2013; Monteiro et al. 2016; Oliveira Maia et al. 2014]. Estudos sugerem a robótica como alternativa para a melhor aprendizagem de diferentes disciplinas, pois a mesma tem se mostrado útil para auxiliar professores e estudantes no processo de ensino aprendizagem de conteúdos desde Computação até outras áreas como Matemática e Física [Pimentel et al. 2015; Rocha et al. 2013].

Segundo Lessa et al. (2015), os estudantes de computação possuem grande interesse pela robótica e automação, pois, estes conteúdos oferecem a possibilidade de materialização daquilo que, até então, era visível somente na tela do computador, permitindo que o aluno reflita, manuseie, construa e execute na prática conceitos abstratos. Assim, a robótica aplicada ao ensino se mostra como uma forma de implementar práticas construtivistas, pois ela permite que o aluno alcance o aprendizado através da busca e investigação [Ribeiro et al. 2011], se mostrando adequada a projetos de ensino, pesquisa e extensão.

O trabalho de Ryan e Deci (2000) citado por Silva, Tedesco e Melo (2014), descreve que motivação representa uma tendência natural para buscar novos desafios e exercitar as próprias capacidades se envolvendo em uma atividade por ser interessante, envolvente ou geradora de satisfação. Nessa perspectiva, projetos que envolvam robótica e automação possuem os elementos necessários para explorar a motivação dos estudantes.

Considerando o exposto, este trabalho descreve a criação e implantação de um grupo de estudo, pesquisa e extensão em robótica e automação como fator motivacional para estudantes de computação utilizando a plataforma de prototipagem de hardware Arduino e o Kit de robótica educacional LEGO Mindstorms. A área definida para a criação do grupo justifica-se pelo fato da robótica e automação possibilitarem que estudantes interajam com o hardware para o qual desenvolvem o software, sendo a lógica inerente na montagem e programação dos robôs e sistemas de automação, envolvendo problemas do mundo real como o da internet das coisas, estimulando assim o aprendizado.

O grupo realiza atividades caracterizadas primordialmente como extensão universitária que consiste em um processo educativo, cultural e científico que articula o ensino e a pesquisa de forma indissociável e viabiliza a relação transformadora entre Universidade e Sociedade [BRASIL 2011]. Assim, a proposta vai de encontro com 
discussões importantes no contexto da indissociabilidade entre ensino-pesquisaextensão, caracterizados como norteadores das universidades brasileiras.

\section{Arduino}

Diante dos objetivos do grupo de estudo, pesquisa e extensão, utilizou-se a placa de prototipagem Arduino. Segundo seu cofundador, o Arduino é uma plataforma de prototipagem de hardware aberta com base em uma placa simples de entrada/saída e um ambiente de desenvolvimento baseado nas linguagens $\mathrm{C} / \mathrm{C}++$. $\mathrm{O}$ Arduino pode ser utilizado para desenvolver objetos interativos independentes, ou conectado a softwares de computador. As placas podem ser montadas manualmente, ou compradas prémontadas [Banzi 2011].

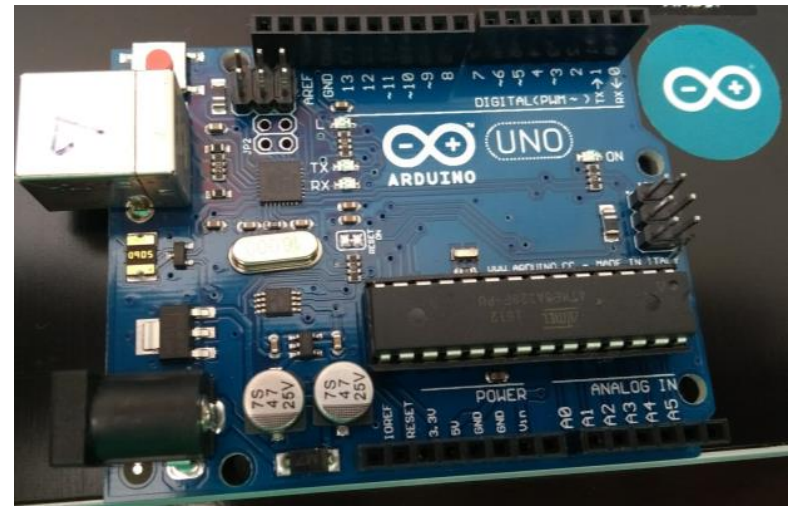

Figura 1. Placa Arduino Uno utilizada pelo grupo

A plataforma de hardware Arduíno é de fácil utilização, permitindo a interação com o usuário através de diferentes componentes eletrônicos como sensores, atuadores e leds. Sensores e atuadores utilizados na plataforma do Arduino aproximam as ideias de algoritmos que utilizam estruturas de repetição e/ou de decisão para resolver problemas específicos da realidade, sendo assim um meio de melhorar a capacidade de percepção dos alunos que determinados passos podem resolver problemas cotidianos, sendo utilizados em projetos de diferentes áreas do conhecimento [Zanetti and Oliveira 2015].

\section{LEGO Mindstorms}

Para inserção de conceitos de robótica, em nosso grupo foi utilizado o kit LEGO Mindstorms NXT 2.0, um kit voltado para a área educacional, o qual permite criar e programar robôs, podendo realizar tarefas simples e complexas. O kit utilizado possui diversas peças para montagens de robôs, como itens estruturais, servo motores, sensores e o brick NXT. O brick NXT é um microprocessador que atua como cérebro do robô. A programação é feita em computadores, pelo uso da linguagem em blocos NXC, já inclusa no kit. Estes aspectos possibilitam ao estudante a montagem de diversos modelos, estimulando a criatividade na resolução de problemas [Baum 2013]. 


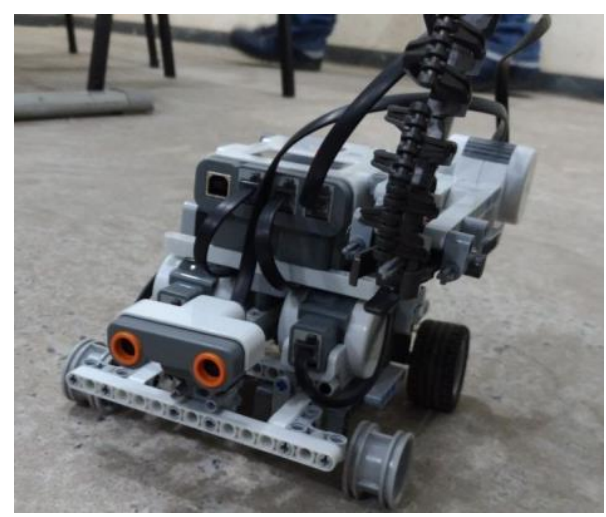

Figura 2. Modelo robótico montado pelo grupo com o Kit LEGO Mindstorms

O Kit de robótica LEGO Mindstorms possui uma grande popularidade, sendo utilizado em diferentes aplicações, não somente na área robótica, mas também para o ensino de conteúdos como computação, automação, física, matemática, entre outros [Aguiar et al. 2015; Maia et al. 2008; Williams 2003].

\section{Trabalhos Relacionados}

Já foram realizados diversos trabalhos que envolvam robótica e automação utilizando a plataforma Arduino e o Kit LEGO Mindstorms em sala de aula, porém relatos relacionando estes tópicos a projetos de extensão são limitados. Nesta sessão são apresentados trabalhos que descrevem as aplicações acadêmicas do kit robótico da LEGO e da plataforma Arduino.

No trabalho de Aguiar et al. (2015), os autores utilizaram do Kit LEGO Mindstorms com vistas a tornar o ensino da programação algo mais simplificado e interessante. Para tanto, os autores realizaram um trabalho com alunos de nível fundamental. Após familiarização com os materiais e softwares, foram ministradas aulas contendo introdução à lógica de programação, utilização de fluxogramas, explicação do kit e atividades de desenvolvimento. Ao concluir as aulas, propuseram um desafio, em que o robô deveria percorrer um trajeto com o uso de sensores de cor e distância, trabalhando todo o conteúdo estudado. O estudo concluiu que a realização do curso foi proveitosa para os alunos do ensino básico. Também se averiguou que o conhecimento dos alunos se nivelou baseado em testes aplicados antes e depois do curso, onde o desvio padrão entre as notas diminuiu. Os autores ainda relataram que em curto prazo, a lógica de programação e a robótica podem auxiliar esses alunos em avaliações que exijam pensamento lógico e abstrato.

Williams (2003) afirma que estudantes tendem a assimilar melhor o que lhes é ensinado quando a informação é entregue de acordo com seu estilo de aprendizagem. Estudantes ativos e sensitivos, ao contrário dos refletivos e intuitivos, aprendem melhor quando precisam utilizar suas mãos e olhos no processo de aprendizagem. Na busca por atender ambas as partes, o autor introduziu a alunos do curso de Engenharia em Computação o ensino da linguagem de programação $\mathrm{C}$ e sistemas embarcados através do Kit LEGO Mindstorms. O objetivo da disciplina foi fazer com que os alunos programassem, em equipes, dois robôs para realizar uma simples tarefa de forma cooperativa. Com este estudo, concluiu-se que os alunos responderam favoravelmente 
ao uso do Kit LEGO Mindstorms no ensino da linguagem C, apontando que $60 \%$ deles recomendariam o uso da ferramenta em usos futuros.

Em trabalho realizado na Universidade de Miami no curso de Sistemas Embarcados [Jamieson 2010], os alunos deveriam escolher entre Arduino, microprocessadores PIC ou placas de prototipagem DE2 FPGA. Mais de 90\% dos alunos escolheram a plataforma Arduino. Os projetos desenvolvidos foram variados, onde fizeram uso de FPGA, Xbox Kinect e interface com um computador. O objetivo principal foi fazer com que os estudantes compreendessem sistemas embarcados e seus vários tópicos e, posteriormente, pudessem projetar estes sistemas. $\mathrm{O}$ trabalho chegou à conclusão que a plataforma Arduino pode ser utilizada para expor os estudantes diferentes tópicos. $\mathrm{O}$ autor ainda aponta que os estudantes puderam escolher a plataforma com qual desejaram trabalhar, o que resultou em uma maior preferência pelo Arduino.

De acordo com Cavalcante et al. (2014) através da tecnologia pode-se instigar o educando a aprender de modo mais eficaz. Em pesquisa desenvolvida pelos autores no Instituto Federal de Educação, Ciência e Tecnologia da Bahia, alunos de graduação em Engenharia Elétrica utilizaram a plataforma Arduino na disciplina de Probabilidade e Estatística. A pesquisa apontou muitas vantagens, tais quais: visualização, plotagem e interpretação de gráficos; realização de cálculos como média, desvio padrão, máximos e mínimos em tempo real; maior ganho de tempo. A pesquisa concluiu que, introduzir e aliar tecnologia com educação é uma ideia factível e de boa aceitação, especialmente tendo em vista seu custo financeiro acessível.

\section{Metodologia}

O grupo de estudo, pesquisa e extensão em Robótica e Automação teve suas atividades iniciadas em maio de 2016 com a participação de oito alunos e ao final do no de 2017 possuía dezenove participantes desenvolvendo atividades caracterizadas em sua maioria como extensão, sendo oito estudantes de nível técnico e onze estudantes de nível superior.

Para implantação do grupo, utilizou-se um conjunto de 4 (quatro) kits de robótica educacional LEGO Mindstorms NXT 2.0 e 20 (vinte) placas de prototipagem de hardware Arduino em conjunto com sensores, atuadores e componentes eletrônicos para elaboração dos projetos. O grupo é composto por alunos dos cursos técnico em Informática integrado ao ensino médio, bacharelado em Sistemas de Informação e Licenciatura em Computação, o qual se reúne semanalmente contemplando uma carga horária mensal em torno de 16 (dezesseis) horas.

Dentre as atividades desenvolvidas pelo grupo pode-se citar: (a) realização de cursos sobre o tema para alunos de escolas publicas e da própria instituição, (b) construção de protótipos para diferentes áreas como automação residencial e agrícola baseado em internet das coisas, (c) criação de modelos de aprendizagem para programação de computadores utilizando o Kit LEGO Mindstorms e a plataforma Arduino, (d) consultoria a outros setores da instituição para automação de processos e captura de dados através de sensores.

Nosso grupo se caracteriza pelo desenvolvimento de atividades que privilegiem a interação aluno-aluno, seja no desenvolvimento de projetos ou enquanto os mesmos 
ministram cursos para a comunidade. Este método de trabalho é importante, pois atividades colaborativas são um dos preditores de maior peso na intenção dos alunos de computação em continuar seu curso superior [Barker et al. 2009].

Para avaliação da proposta, realizou-se um levantamento qualitativo com os alunos participantes do grupo utilizando-se a metodologia de avaliação de objetos de aprendizagem descrita por Savi et al. (2010) a qual é baseada na taxonomia de Bloom para avaliação da aprendizagem [Bloom et al. 1984] e no modelo de Keller para avaliação da motivação [Keller 2009]. Este instrumento permite auxiliar a avaliação de objetos de aprendizagem e aquisição do conhecimento através de um questionário para mensurar itens como (a) atenção, (b) compreensão, (c) divertimento, (d) desafio e (e) motivação com objetivo de obter dados relacionados ao incremento da motivação e aprendizado dos alunos participantes do grupo.

Assim, tendo em vista o objetivo de mensurar o envolvimento dos estudantes e seus sentimentos de confiança e motivação acerca dos conteúdos da área de computação, automação e robótica, foi elaborado um questionário contendo 15 questões baseadas na escala de Likert de 1 a 5 (1 - para Discordo Totalmente; 5 - para Concordo Totalmente), e uma questão do tipo verdadeira ou falsa. $\mathrm{O}$ questionário aplicado e a divisão nas dimensões de análise dos resultados estão acessíveis pela url https://goo.gl/pvvs7D.

\section{Resultados e Discussões}

Na Figura 3 são apresentados alguns trabalhos desenvolvidos pelos integrantes do grupo. Na url https://goo.gl/photos/L3DXHGMM8JVfGrpJ7 são apresentadas algumas fotos de atividades desenvolvidas.

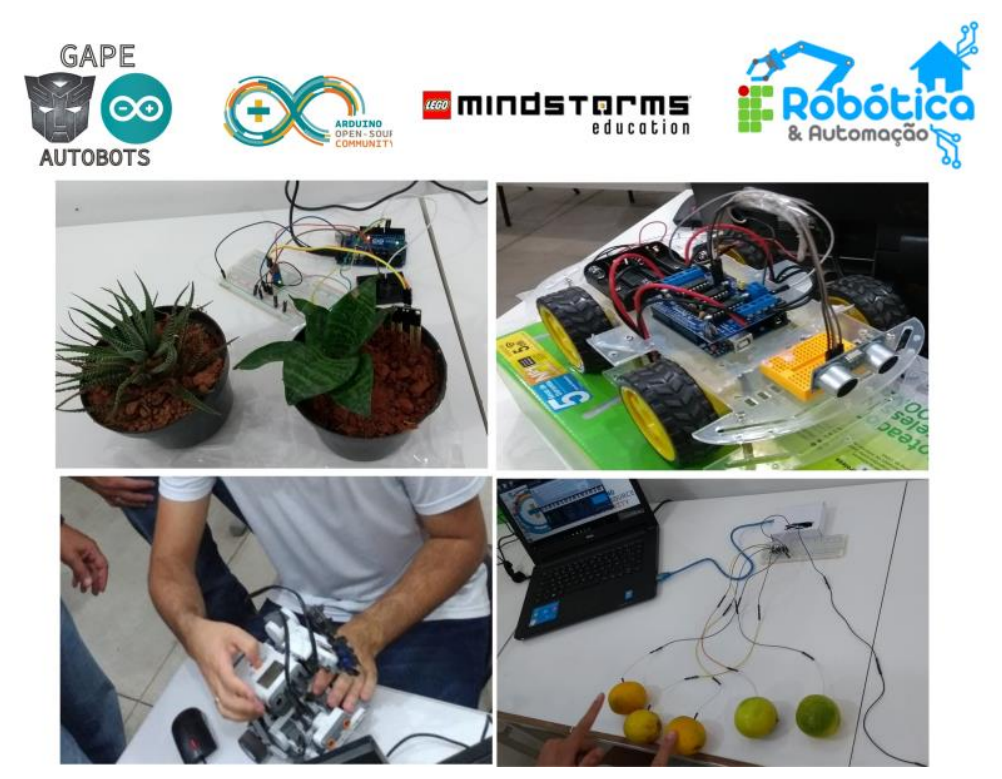

Figura 3. Trabalhos desenvolvidos pelo grupo

Ao final de cada semestre letivo os estudantes participantes recebem um certificado o qual podem contabilizar como atividades complementares. Tendo isto em vista, os mesmos foram questionados se participam do grupo por necessidade de algum 
certificado. Como se pode observar na Figura 4, apenas 5\% (correspondente a um aluno) respondeu que participa por este motivo, o que indica que os mesmos participam do grupo por buscar novos desafios e para exercitar as próprias capacidades, algo descrito por Ryan e Deci (2000) e observado na alta concordância com as dimensões de desafio e compreensão observados na Figura 5.

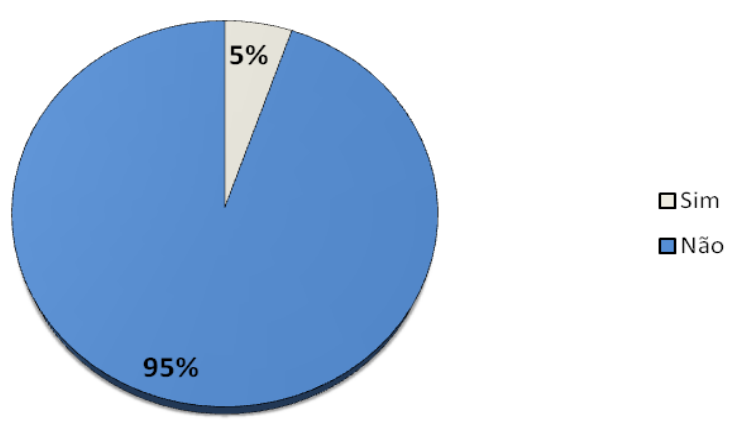

Figura 4. Gráfico de concordância com a afirmação "Participo do grupo por alguma imposição ou necessidade de certificados"

O gráfico apresentado na Figura 5 demonstra a distribuição da intensidade de concordância atribuída pelos estudantes a cada uma das dimensões avaliadas.

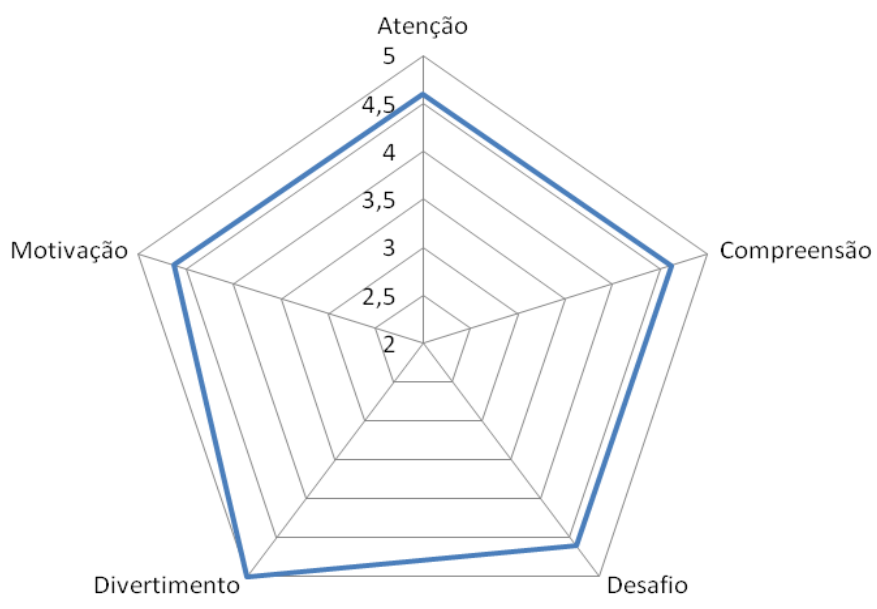

Figura 5. Gráfico radar das dimensões avaliadas pelos estudantes participantes do grupo

A partir dos resultados obtidos, observou-se uma alta concordância dos estudantes com as questões apresentadas, destacando-se a concordância máxima para as questões da dimensão de divertimento, o que vai de encontro com o trabalho de Somyürek (2015) o qual descreve em sua pesquisa que a utilização de kits robóticos oferece oportunidades para aprofundar a compreensão dos alunos de vários conceitos com exploração prática resultando em diversão e prazer.

Ademais, cabe-se destaque a uma questão avaliada na dimensão de motivação dos estudantes tendo em vista os objetivos deste trabalho. Para a questão "Participar deste grupo me motiva a permanecer na área de computação /automação/robótica", 
houve um nível de concordância máxima por parte de $74 \%$ dos estudantes, outros $21 \%$ apresentaram uma concordância de intensidade 4 (quatro), enquanto apenas um estudante $(5 \%)$ não apresentou concordância com a afirmação (Figura 6). O cenário obtido corrobora com Barker et al. (2009) que descreve em seu trabalho que o desenvolvimento de atividades práticas que privilegiam a interação aluno-aluno são preditores na motivação dos mesmos em continuar um curso na área de computação.

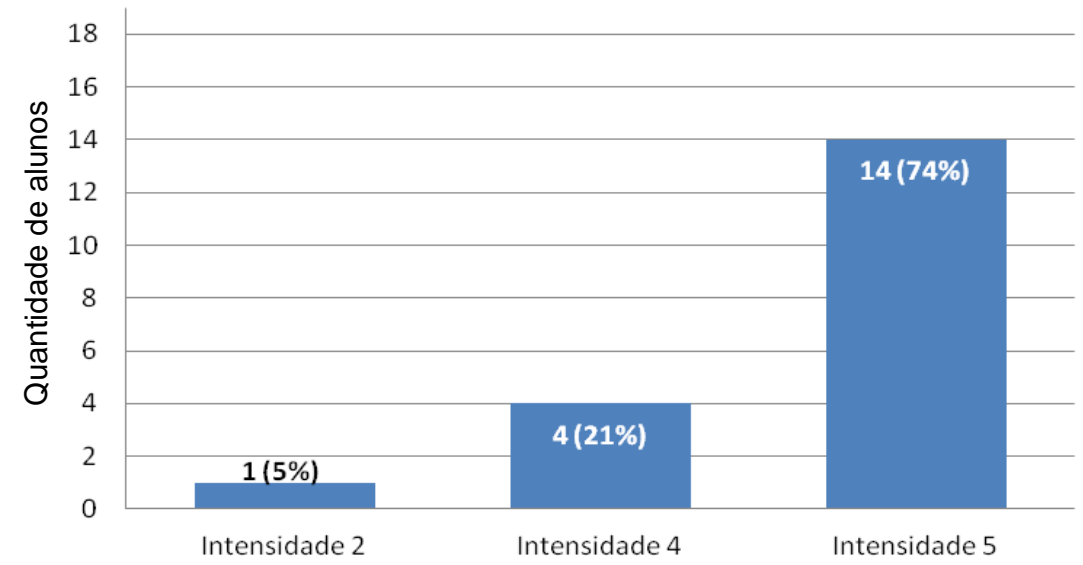

Figura 6. Gráfico de concordância com a afirmação "Participar deste grupo me motiva a permanecer na área de computação /automação/robótica"

\section{Considerações Finais}

Diante dos resultados obtidos e atividades desenvolvidas, constatou-se que a abordagem proposta tem, de fato, capacidade de potencializar a motivação dos estudantes de computação, não somente no ensino superior, mas também no ensino técnico integrado. $\mathrm{O}$ fator motivacional e de divertimento manifestados pelos alunos foram preponderantes dentre os aspectos que confirmam a capacidade da proposta. Além de se sentirem motivados, os educandos possuem a oportunidade de externar sua criatividade com a plataforma de hardware Arduino e o kit robótico da LEGO, seja em trabalhos propostos, cursos ministrados ou em projetos independentes.

Nossa avaliação sobre o grupo de estudo, pesquisa e extensão ainda é limitada tendo em vista o caráter experimental da proposta e a população pequena analisada, porém os dados obtidos demonstram que este pode ser um recurso interessante a se desenvolver nas comunidades acadêmicas de computação. Com a inclusão de inovações tecnológicas, como conteúdos relacionados à internet das coisas, o estudante procura com maior empenho o conhecimento e se interessa em participar mais daquilo que the foi proposto, seja por curiosidade, por necessidade em sua área profissional ou, puramente, para sua diversão.

\section{Agradecimentos}

Ao Instituto Federal de Educação, Ciência e Tecnologia do Sul de Minas Gerais IFSULDEMINAS pelo apoio concedido. 


\section{Referências}

Aguiar, Y. Q., Maciel, B. K., Matos, S. D. G., Soares, L. B. and Oliveira, V. M. (2015). Introdução à Robótica e Estímulo à Lógica de Programação no Ensino Básico Utilizando o Kit Educativo LEGO ${ }^{\circledR}$ Mindstorms. In Anais dos Workshops do CBIE.

Banzi, M. (2011). Primeiros passos com o Arduino. São Paulo: Novatec, p. p1.

Barker, L. J., McDowell, C. and Kalahar, K. (2009). Exploring factors that influence computer science introductory course students to persist in the major. In $A C M$ SIGCSE Bulletin. ACM.

Baum, D. (2013). Dave Baum's Definitive Guide to Lego Mindstorms. APress.

Bloom, B. S., Krathwohl, D. R. and Masia, B. B. (1984). Bloom taxonomy of educational objectives. Pearson Education.

BRASIL (2011). Plano Nacional de Extensão Universitária.

Cavalcante, M. M., Silva, J. L. de S., Viana, E. C. and Dantas, J. R. (2014). A plataforma Arduino para fins didáticos: estudo de caso com recolhimento de dados a partir do PLX-DAQ. In XXXIV Congresso da Sociedade Brasileira de Computação (CSBC 2014), Brasília, DF.

Cisco (2016). The Network Skills in Latin America - White Paper.

Correll, N., Wing, R. and Coleman, D. (2013). A one-year introductory robotics curriculum for computer science upperclassmen. IEEE Transactions on Education, v. 56, n. 1, p. 54-60.

Csikszentmihalyi, M. and Wong, M. M. (2014). Motivation and academic achievement: The effects of personality traits and the quality of experience. Applications of flow in human development and education. Springer. p. 437-465.

Felder, R. M. and Silverman, L. K. (1988). Learning and teaching styles in engineering education. Engineering education, v. 78, n. 7, p. 674-681.

Hoed, R. M. (2017). Análise da evasão em cursos superiores: o caso da evasão em cursos superiores da área de computação - Dissertação (mestrado) - UNB.

INEP (2014). Censo da Educação Superior - Resumo Técnico.

Jamieson, P. (2010). Arduino for teaching embedded systems. are computer scientists and engineering educators missing the boat? Proc. FECS, p. 289-294.

Keller, J. M. (2009). Motivational design for learning and performance: The ARCS model approach. Springer Science \& Business Media.

Lessa, V., Forigo, F., Teixeira, A. and Licks, G. P. (2015). Programação de Computadores e Robótica Educativa na Escola: tendências evidenciadas nas produções do Workshop de Informática na Escola. In Anais do Workshop de Informática na Escola. CBIE.

Maia, L. D. O., Da Silva, V. J., Rosa, R. E. V. de S., De Lucena Junior, V. F. and De Queiroz Neto, J. P. (2008). A Robótica como Ambiente de Programaç ao Utilizando o Kit Lego Mindstorms. In Simposio Brasileiro de Informática na Educação - SBIE.

Monteiro, D., Bremgartner, V., Lima, H. and Salgado, N. (2016). Uma Experiência do 
Uso Do Hardware Livre Arduino no Ensino De Programação De Computadores. In Anais do Workshop de Informática na Escola. CBIE.

Oliveira Maia, R., Da Silva, F. A., Pazoti, M. A., De Almeida, L. L. and Pereira, D. R. (2014). DESENVOLVIMENTO DE UM DISPOSITIVO PARA APOIO AO ENSINO DE COMPUTAÇÃO E ROBÓTICA. Colloquium Exactarum, v. 6, n. 2.

Pimentel, C., Sampaio, F. and Revoredo, K. (2015). Mecatrônica educacional apoiando o aprendizado de conceitos de física e matemática: Um estudo de caso. In Anais do Workshop de Informática na Escola. . CBIE.

Ribeiro, P. C., Martins, C. B. and Bernardini, F. C. (2011). A Robótica como Ferramenta de Apoio ao Ensino de Disciplinas de Programação em Cursos de Computação e Engenharia. In Anais do Workshop de Informática na Escola. CBIE.

Rocha, F. S., Maranghello, G. F. and Lucchese, M. M. (2013). Acelerômetro eletrônico e a placa Arduino para ensino de física em tempo real. Caderno Brasileiro de Ensino de Física, v. 31, n. 1, p. 98-123.

Ryan, R. M. and Deci, E. L. (2000). Intrinsic and extrinsic motivations: Classic definitions and new directions. Contemporary educational psychology, v. 25, n. 1, p. $54-67$.

Savi, R., Von Wangenheim, C. G., Ulbricht, V. and Vanzin, T. (2010). Proposta de um modelo de avaliação de jogos educacionais. RENOTE, v. 8, n. 3.

Silva, T. S. C., Tedesco, P. C. and Melo, J. C. B. (2014). A importância da motivação dos estudantes e o uso de técnicas de engajamento para apoiar a escolha de jogos no ensino de programação. In Simpósio Brasileiro de Informática na Educação.

Somyürek, S. (2015). An effective educational tool: construction kits for fun and meaningful learning. International Journal of Technology and Design Education, v. 25 , n. 1, p. 25-41.

Williams, A. B. (2003). The qualitative impact of using LEGO MINDSTORMS robots to teach computer engineering. IEEE Transactions on Education, v. 46, n. 1, p. 206.

Zanetti, H. and Oliveira, C. (2015). Práticas de ensino de Programação de Computadores com Robótica Pedagógica e aplicação de Pensamento Computacional. In Congresso Brasileiro de Informática na Educação. 